\section{Bell Curve}

Ericka L. Wodka

Center for Autism and Related Disorders, Kennedy Krieger Institute and The Johns Hopkins University School of Medicine, Baltimore, MD, USA

\section{Synonyms}

Gaussian distribution; Normal curve; Normal distribution

\section{Definition}

A normal distribution of observations/scores is shaped like a "bell," with the majority of observations/scores occurring around the mean and increasingly fewer observations/scores occurring farther (above/below) from the mean $(68.26 \%$ of observations/scores fall within one standard deviation of the mean; $95.44 \%$ fall within two standard deviations of the mean). A normal distribution of observations is typical in large samples acting additively and independently and is assumed in parametric statistics (e.g., t-tests, ANOVA). Standardized scores derived from neuropsychological measures are based upon (assume) normal distribution of the standardization sample. While this assumption provides a common metric that allows for direct comparison of performance between different measures, it is important to note that score distributions for a number of neuropsychological tests are non-normal (e.g., Boston Naming Test, Wisconsin Card Sorting Test, Mini-Mental Status Exam, Test of Memory Malingering). For this reason, selection of measures and interpretation of test findings must include consideration of score distributions (Strauss et al. 2006).

\section{Cross-References}

- Base Rate (Population)

$\checkmark$ Cut Off Scores, Cutting Scores

$\checkmark$ Intelligence Quotient

- Mental Age

- Percentiles

- Standard Scores

\section{References and Readings}

Lezak, M. D., Howieson, D. B., Bigler, E. D., \& Tranel, D. (2012). Neuropsychological assessment (5th ed.). New York: Oxford University Press.

Sattler, J. M. (2001). Assessment of children: Cognitive foundations (5th ed.). La Mesa: Jerome M. Sattler.

Strauss, E., Sherman, E. M. S., \& Spreen, O. (2006). A compendium of neuropsychological tests: Administration, norms, and commentary (3rd ed., pp. 3-43). New York: Oxford University Press. 